\title{
Cigarette smoking leads to persistent and dose- dependent alterations of brain activity and connectivity in anterior insula and anterior cingulate
}

\author{
Davide Zanchi', Arthur L. Brody ${ }^{2,3}$, Marie-Louise Montandon', Rotem Kopel ${ }^{1,4}$, Kirsten Emmert', \\ Maria Giulia Preti ${ }^{1,4}$, Dimitri Van De Ville ${ }^{1,4}$ \& Sven Haller $5,6,7,8$ \\ Department of Imaging and Medical Informatics, University Hospitals of Geneva and Faculty of Medicine of the University of Geneva, Switzerland', Department of \\ Psychiatry, University of California at Los Angeles ${ }^{2}$, Departments of Psychiatry and Research, VA Greater Los Angeles Healthcare System ${ }^{3}$, Institute of \\ Bioengineering, Ecole Polytechnique Fédérale de Lausanne, Switzerland ${ }^{4}$, Faculty of Medicine of the University of Geneva, Switzerland ${ }^{5}$, Department of Surgical \\ Sciences, Radiology, Uppsala University, Uppsala, Sweden ${ }^{6}$, Department of Neuroradiology, University Hospital Freiburg, Germany ${ }^{7}$ and Affidea Centre de \\ Diagnostic Radiologique de Carouge CDRC, Geneva, Switzerland ${ }^{8}$
}

\begin{abstract}
Although many smokers try to quit smoking, only about 20-25 percent will achieve abstinence despite 6 months or more of gold-standard treatment. This low success rate suggests long-term changes in the brain related to smoking, which remain poorly understood. We compared ex-smokers to both active smokers and non-smokers using functional magnetic resonance imaging (fMRI) to explore persistent modifications in brain activity and network organization. This prospective and consecutive study includes 18 non-smokers ( $29.5 \pm 6.7$ years of age, 11 women), 14 smokers ( $\geq 10$ cigarettes a day $>2$ years of smoking, $29.3 \pm 6.0$ years of age, 10 women) and 14 ex-smokers ( $>1$ year of quitting $30.5 \pm 5.7$ years of age, 10 women). Participants underwent a block-design fMRI study contrasting smoking cue with control (neutral cue) videos. Data analyses included task-related general linear model, seed-based functional connectivity, voxel-based morphometry (VBM) of gray matter and tract-based spatial statistics (TBSS) of white matter. Smoking cue videos versus control videos activated the right anterior insula in ex-smokers compared with smokers, an effect correlating with cumulative nicotine intake (pack-years). Moreover, ex-smokers had a persistent decrease in functional connectivity between right anterior insula and anterior cingulate cortex (ACC) compared with control participants, but similar to active smokers. Potentially confounding alterations in gray or white matter were excluded in VBM and TBSS analyses. In summary, ex-smokers with long-term nicotine abstinence have persistent and dosedependent brain network changes notably in the right anterior insula and its connection to the ACC.
\end{abstract}

Keywords Craving, nicotine, smoking.

Correspondence to: Sven Haller, Affidea Centre de Diagnostic Radiologique de Carouge CDRC, Geneva, Switzerland, Department of Surgical Sciences, Radiology, Uppsala University, Uppsala, Sweden, Department of Neuroradiology, University Hospital Freiburg, Germany, Faculty of Medicine of the University of Geneva, Switzerland. E-mail: sven.haller@me.com

\section{INTRODUCTION}

Smoking is considered one of the largest single causes of preventable death (OECD 2012a, Online, OECD Publishing, Paris, available at: www.oecd.org/health/). About 23 percent of the European population smokes cigarettes, with about 31 percent of this population having tried to quit within the last 12 months ("European Union Campaign 2013: Ex-smokers are Unstoppable"). The relapse rate in the first month of unaided abstinence is about 80 percent, and only 3-5 percent of untreated patients remain abstinent after 6 months (Polosa et al. 2011; Gualano et al.
2014). Furthermore, only about 20-25 percent of smokers will achieve abstinence with six months or more of goldstandard treatment (Hurt et al. 1997; Hughes et al. 1999; Jorenby et al. 1999; Killen et al. 1999; Holmes et al. 2004, 2004). These observations suggest that smoking leads to persistent modifications in the brain that contribute to high relapse rates (DeBry \& Tiffany 2008), an area of research which remains largely unexplored. Although several studies have been conducted on nicotine addiction and short-term nicotine abstinence to understand neurobiological mechanisms underlying smoking craving, further investigations are needed to determine the effects of long-term nicotine 
abstinence and smoking cessation in order to ultimately better prevent smoking relapse ("Clearing the smoke," 2014).

In the field of neuroimaging, several functional magnetic resonance imaging (fMRI) studies focused on differences in brain activity between control and active smokers who were abstinent for relatively short periods of time (up to 24 hours) to investigate the neurobiological mechanisms underlying nicotine abstinence and smoking addiction (Carroll et al. 2013; Ding \& Lee 2013; Sweitzer et al. 2013; Geier et al. 2014). These findings have indicated a network of brain regions that underlie craving in addiction in general (Luigjes et al. 2012), with cravingrelated information being processed in the orbitofrontal cortex (OFC), anterior cingulate cortex (ACC) and nucleus accumbens. Furthermore, the hippocampus, insula and amygdala were found to be responsible for modulating craving by their functional connectivity to the aforementioned regions (George \& Koob 2013).

While these studies identified key brain regions involved in addiction and short-term tobacco withdrawal, there is a lack of knowledge about which brain areas underlie long-term nicotine abstinence. To date, only one fMRI investigation included ex-smokers and demonstrated that greater prefrontal cortical activation (in response to an attentional bias paradigm and during error monitoring in a go/non-go paradigm) might be a characteristic of smokers who are successful in maintaining abstinence (Nestor et al. 2011). These findings point towards a general pattern of increased prefrontal cortical activity in ex-smokers suggesting that top-down control might be an important aspect of long-term nicotine abstinence.

Given these prior findings, we assessed changes in brain activity and connectivity in ex-smokers with long-term tobacco cessation, with the aim of identifying long-term persistent modification in brain activation related to cue-induced craving. To this end, we used fMRI in a task-based paradigm with smoking-related videos in three populations: ex-smokers (more than 1 year of smoking abstinence); active smokers; and non-smokers. We sought to determine if persistent modifications of brain activity occur in ex-smokers, and whether these modifications were dependent on pack-years of smoking history (a potential marker of cumulative nicotine exposure and lifetime cue-conditioning between cigarettes and nicotine). In addition, we also sought to determine changes in functional connectivity in ex-smokers.

\section{MATERIALS AND METHODS}

\section{Participants}

The local institutional ethical committee approved this prospective and consecutive study. All participants gave written informed consent prior to inclusion. Forty-six subjects were recruited through local and Internet advertising. Inclusion criteria for ex-smokers were abstinence from cigarettes for more than 12 months prior to the study and previous smoking period of at least 2 years with 10 or more cigarettes per day. Inclusion criteria for smokers were smoking 10 or more cigarettes per day over at least 2 years, no intention to quit smoking in the next month and a period of 15 minutes before the study without smoking to have a uniform period of abstinence prior to scanning. Inclusion criteria for non-smokers were no regular tobacco use in their lifetime and no intention to start smoking in the next months. The three groups were matched for gender and age, with the ex-smokers and the smokers groups matched also for years of smoking and number of cigarettes smoked per day. Exclusion criteria for all participants were history of drug or alcohol abuse/dependence, major medical disorders and use of medications that could affect brain function (e.g. psychotropics, stimulants or $\beta$-blockers) on a regular basis. Exsmokers were additionally considered ineligible if they reported recent or current use of products to facilitate nicotine abstinence (e.g. gum, patch, lozenge, nasal spray or inhaler).

The final sample included 14 ex-smokers ( $>1$ year of abstinence, $30.5 \pm 5.7$ years of smoking, 10 women), 14 smokers $(\geq 10$ cigarettes a day for $>2$ years, 29.3 \pm 6.0 years of smoking, 10 women) and 18 non-smokers $(29.5 \pm 6.7$ years, 11 women) (Table 1$)$. Ex-smokers had a non-significant trend towards an earlier age of first use (18.5 \pm 1.1 years) as compared with current smokers $20.7 \pm 0.8$ years $)$.

\section{Functional magnetic resonance imaging acquisition}

Images were obtained using a 3T scanner (Trio; Siemens, Erlangen, Germany) with a standard 32 channel headcoil. Functional MRI of the whole brain was acquired by echo planar imaging using the following parameters: whole brain coverage, $96 \times 96$ matrix, $\mathrm{TR}=2.5 \mathrm{~s}$, $\mathrm{TE}=30 \mathrm{~ms}, 34$ slices, and 245 repetitions. In addition,

Table 1 Essential demographic parameters of the included study groups.

\begin{tabular}{|c|c|c|c|c|}
\hline & \multicolumn{4}{|c|}{ Demographic variables } \\
\hline & Non-smokers & Ex-smokers & Smokers & Test \\
\hline Cases & 18 & 14 & 14 & \\
\hline Females & 11 & 10 & 10 & $\mathrm{n} / \mathrm{s}$ \\
\hline Age & $29.5(6.7)$ & $30.5(5.7)$ & $29.3(6.0)$ & $\mathrm{n} / \mathrm{s}$ \\
\hline Year of Smoking & $8.1(4.9)$ & $8.6(4.5)$ & $\mathrm{n} / \mathrm{s}$ & \\
\hline Cigarettes per day & $13.3(3.4)$ & $12.7(3.1)$ & $\mathrm{n} / \mathrm{s}$ & \\
\hline Years since quitting & $3.7(3.0)$ & & & \\
\hline
\end{tabular}

Values represent mean (standard deviation). 
a 3D T1-weighted structural scan $(256 \times 256$ matrix size, 176 sections, $1 \times 1 \times 1 \mathrm{~mm}^{3}, \quad \mathrm{TE}=2.3 \mathrm{~ms}$, $\mathrm{TR}=2300 \mathrm{~ms}$ ) and diffusion tensor imaging (DTI) scan (30 diffusion directions $b=1000 \mathrm{~s} / \mathrm{mm}^{2}$ isotropically distributed on a sphere, 1 reference $b=0 \mathrm{~s} / \mathrm{mm}^{2}$ image with no diffusion weighting, $128 \times 64$ matrix, $2 \times 2 \times 2 \mathrm{~mm}$ voxel size, $\mathrm{TE}=92 \mathrm{~ms}, \mathrm{TR}=9000 \mathrm{~ms}$ and 1 average) were acquired.

\section{Functional magnetic resonance imaging procedure}

Participants underwent fMRI between 3 and 6 PM to control for potential effects due to time of day. The paradigm consisted of an on-off block-design with two active conditions (smoking cue and control videos) and a neutral condition (cross displayed). The active condition used video cues developed by Brody et al. (Brody et al. 2007; Culbertson et al. 2011). These cues were filmed from the first person point of view and were 45 seconds in length. Each smoking video shows a potential craving situation, such as writing a letter and smoking a cigarette or standing outside of a nightclub smoking a cigarette. The control videos were matched for similar content except for the absence of smoking cues. After each video, a visual analog scale was presented for 2.5 seconds, and participants rated the degree of craving using an MR-compatible response box. The rating scale included seven steps from no craving to high craving. After the rating, a rest period consisted of the visual presentation of a fixation cross for 10 seconds. Each run included five smoking and five control videos in a pseudo-randomized fashion and lasted 612 seconds. Each participant performed two runs. Before fMRI scanning, participants were instructed on the procedure and became familiar with the task by a training run outside of the MRI scanner.

\section{Statistical analysis}

Statistical analyses were conducted using GraphPad Prism (Version 6, GraphPad Software, San Diego, CA, USA), Matlab (Version 2014a, The MathWorks Inc., Natick, MA, USA) and FSL (Version 5.0.6, FMRIB, Oxford, UK).

\section{Analysis of demographic and behavioral data}

Normally distributed variables, notably age and years of smoking, were analyzed using an analysis of variance (ANOVA) with Tukey correction for post hoc pairwise comparisons. Non-normally distributed variables, notably gender, number of cigarettes a day and rating scores, were tested using Kruskal-Wallis nonparametric test with Dunn's correction for post hoc pairwise comparisons.

\section{Task-related general linear model}

Processing and analysis of imaging data were performed using FSL FEAT (fMRI Expert Analysis Tool version 6.00, http://fsl.fmrib.ox.ac.uk/fsl/fslwiki/FEAT). Preprocessing included brain extraction using FSL's Brain Extraction Tool (BET), motion correction using FSL's MCFLIRT (intra-modal motion correction tool) (Jenkinson et al. 2002) and smoothing using FSL's SUSAN (noise reduction uses nonlinear filtering) (Smith et al. 1997). In addition, for each subject, we computed a maximum of the framewise displacement (Dijk Van, Sabuncu, \& Buckner 2012; Power et al. 2012) from the realignment parameters and subjected this to group comparison (ANOVA).

The linear-model analysis included three levels. At the first level, the contrast 'smoking versus control videos' was calculated separately for each run of each participant using fixed-effects analysis. Then, at the second level, one fixed-effects analysis was conducted that included both runs of each subject. Finally, at the third level, a random-effects analysis was performed to investigate group differences between ex-smokers, smokers and non-smokers. This resulted in a mixed-effects group model implementing FLAME 1 (FMRIB's Local Analysis of Mixed Effects). Finally, a permutation-based nonparametric test (randomize, FSL tool) was applied, correcting for multiple comparisons by threshold-free cluster enhancement (Winkler et al. 2014). $P$ values $<0.05$ were considered as significant.

\section{Correlation between imaging data and pack-year smoking history}

Additionally, we tested possible correlations between regions of the brain that were significantly activated in the contrast of ex-smokers versus smokers (notably the right anterior insula (raINS)) and the accumulated nicotine dose estimated by pack-year smoking history.

\section{Functional connectivity analysis}

Based on results of the task-related general linear model (GLM) and the previous literature on ex-smokers (Nestor et al. 2011), we defined regional masks and extracted region-averaged time-courses of each subject for the ACC, raINS and left frontal pole (FP). Functional connectivity analysis was performed using a custom-built toolbox in Matlab. In particular, for each subject, we computed all pairwise correlations (3) between the time-courses of these regions to estimate functional connectivity. These values were then submitted, for each connection, first to a one-sample $t$-test across subjects (to assess significant connectivity) and subsequently to an ANOVA (to assess group differences) with Tukey correction for post hoc pairwise comparisons. 
Voxel-based morphometry analysis of T1 images

FSL software was used to perform the voxel-based morphometry (VBM) analysis (http://fsl.fmrib.ox.ac.uk) to assess for gray matter (GM) density differences between groups. Standard processing steps were used (Smith et al. 2006, 2007). In particular, first brain extraction and tissue-type segmentation were conducted using the corresponding FSL tools (BET and FAST4). A non-linear transformation into Montreal Neurological Institute reference space was applied and a study-specific GM template was created. All the native GM images were then non-linearly registered to this template. Later on, the images were smoothed with an isotropic Gaussian kernel of $2 \mathrm{~mm}$ sigma. The same non-parametric test was applied as for the analysis of the task-related GLM and $P$ values $<0.05$ were considered as significant.

Tract-based spatial statistics analysis of diffusion tensor imaging data

As for the VBM, FSL software was used to analyze DTI data, according to the standard procedure (Smith et al. 2004) to test for white matter (WM) integrity differences between groups. First, by a non-linear registration, all subjects' fractional anisotropy data were projected onto a mean fractional anisotropy tract skeleton. Later, by using a non-linear registration, voxelwise statistical analysis with threshold-free cluster enhancement correction for multiple comparisons was performed, considering TFCE-corrected $P$ values $<0.05$ as significant.

\section{RESULTS}

Rating scores

Smoking videos induced higher craving in smokers than non-smokers $(P<0.01)$ or ex-smokers $(P<0.05)$ as well as for ex-smokers than non-smokers $(P<0.05)$ (Fig. 1). There were no significant group differences for control videos concerning both the raw difference and the relative difference between smoking and control videos to compensate for inter-individual differences in the rating.

\section{Task-related general linear model}

From group analyses of framewise displacement, no significant effect of motion was found between the groups.

In the task-related GLM, we considered the contrast of 'smoking videos versus control videos'. The comparison 'ex-smokers versus smokers' revealed significantly greater activations in ex-smokers in the raINS, right frontal operculum and right inferior frontal gyrus (IFG) (Fig. 2a and Supporting Information Supplementary Table A).

The comparison 'ex-smokers versus non-smokers' revealed a significantly greater activation in ex-

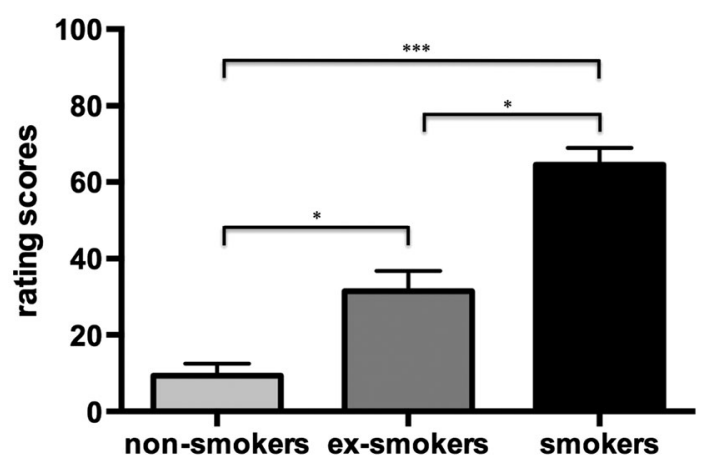

Figure I Rating scores for smoking videos. The rating scores for craving during smoking cue videos $(0=$ no craving, $100=$ maximum craving). Active smokers had significantly higher craving than exsmokers $(P<0.05)$ and non-smokers $(P<0.001)$. Ex-smokers had higher craving than non-smokers $(P<0.05)$. The barplots indicate mean values and standard error. ${ }^{*} P<0.05$, **** $P<0.001$

smokers bilaterally in the FP, (IFG) and bilateral anterior insula (aINS) with a higher activation in the raINS (Fig. 2b).

The comparison 'smokers versus non-smokers' revealed increased activation in the ACC and in the cerebellum (Fig. 2c). There were no de-activations in the aforementioned contrasts (Supporting Information Supplementary Figure A).

Correlation between imaging data and accumulated cigarettes consumption

A positive correlation $(P<0.05)$ was found in the ex-smokers group between the cumulative cigarette dose (i.e. measured in pack-years) and the activation strength in the raINS (Fig. 3).

\section{Functional connectivity}

The one-sample $t$-tests showed significant effect of connectivity for all connections $(P<0.0001)$. Only the ANOVA performed on the connection raINS-ACC showed a significant group difference $(P<0.01)$. For this connection, post hoc tests revealed statistical differences between non-smokers and smokers $(P<0.01)$, and between non-smokers and ex-smokers $(P<0.05)$. No significant differences were found for the comparison between smokers and ex-smokers (Fig. 4).

\section{Voxel-based morphometry and tract-based spatial} statistics analysis

The VBM analysis of GM density and the tract-based spatial statistics (TBSS) analysis of WM revealed no statistical differences between study groups. 
Figure 2 Task-related general linear model analyses for the comparison of smoking videos versus control videos. Ex-smokers versus smokers (a) had increased activations notably in the right anterior insula, frontal operculum and inferior frontal gyrus. Ex-smokers versus non-smokers (b) had increased activations notably in the bilateral frontal pole inferior frontal gyrus and right anterior insula. Smokers versus nonsmokers (c) had higher activations notably in the ventral anterior cingulate cortex. The inverse comparisons of smokers versus ex-smokers, nonsmokers versus ex-smokers and nonsmokers versus smokers yielded no supra-threshold activations. Activations are superimposed on the template brain in Montreal Neurological Institute space, cluster threshold at $Z=2.3$ corresponding to $P=0.05$ (corrected)

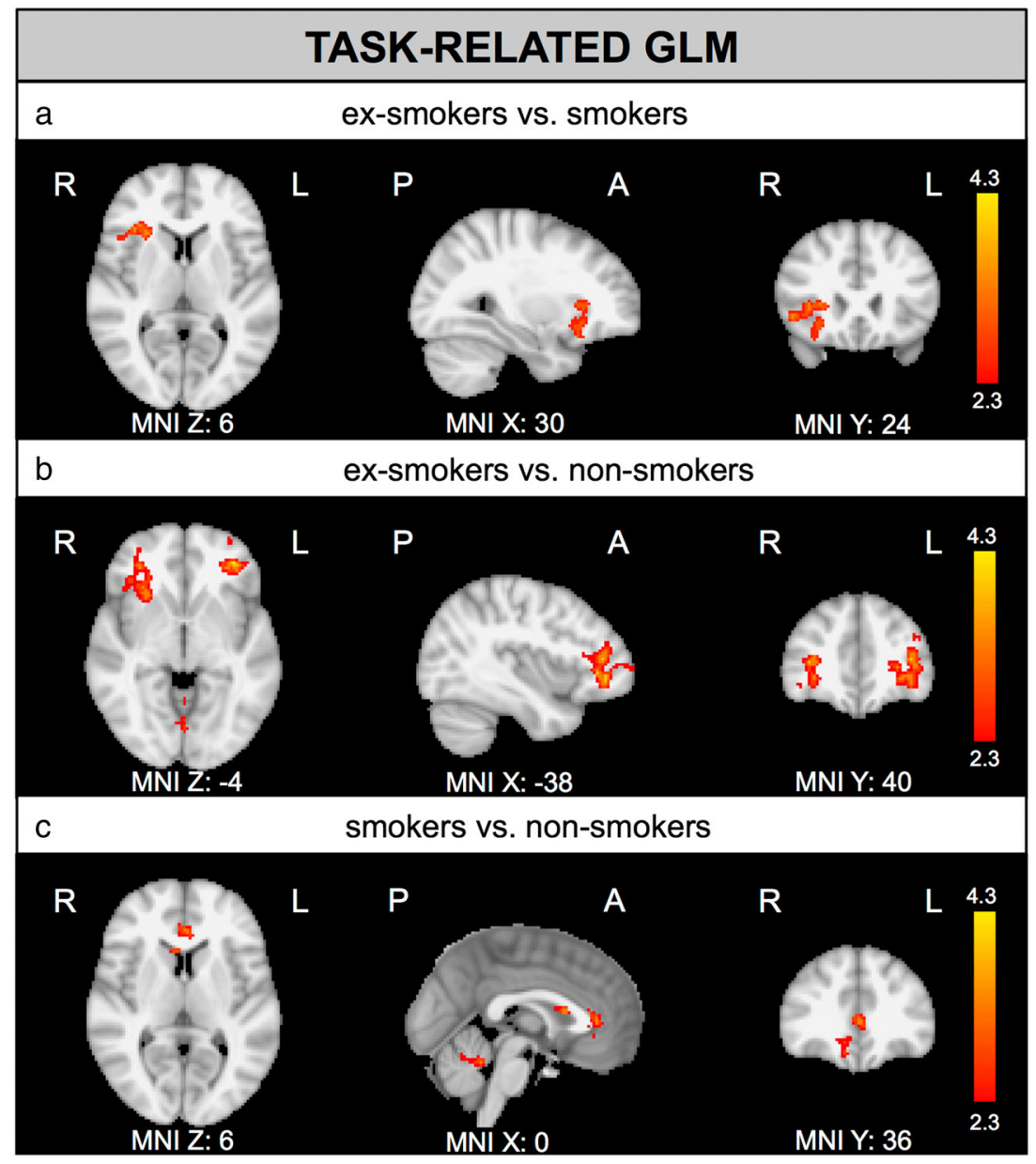

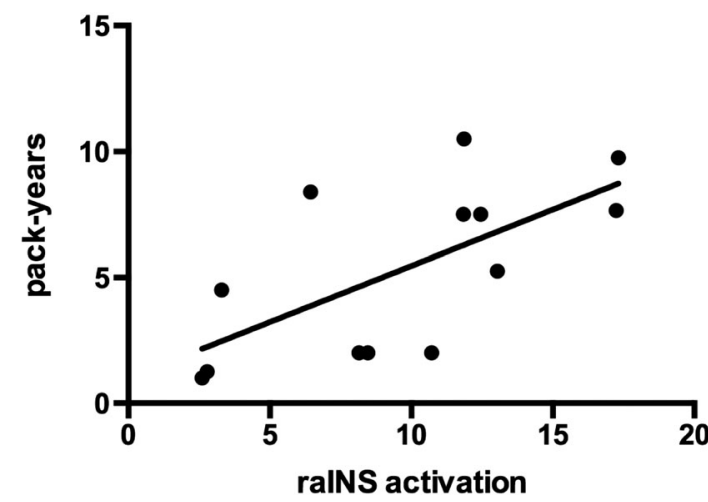

Figure 3 Correlation between nicotine dose and functional magnetic resonance imaging (fMRI) activation in right anterior insula in ex-smokers. Correlation in ex-smokers between the accumulated nicotine dose (measured in pack-years) and the $\mathrm{fMRI}$ activation strength in the right anterior insula measured as contrast of parameter estimates for the contrast of smoking cue videos versus control videos, which was significant at $P<0.05$

\section{DISCUSSION}

The current study aims at providing new insights into neural mechanisms that underlie long-term cigarette abstinence in ex-smokers. Three groups of participants, ex-smokers, active smokers and control non-smokers, underwent fMRI during presentation of smoking cue videos and control videos, which were previously used and validated in fMRI studies comparing active smokers versus control participants (Brody et al. 2007; Culbertson et al. 2011).

Our first finding relates to task-related brain activity during smoking versus control cue videos. The difference between active smokers and non-smokers were in line with previous results that showed increased activation in ACC induced by smoking-related stimuli (Brody et al. 2007; Wang et al. 2007; Azizian et al. 2010). However, the contrast between ex-smokers and non-smokers showed increased activation in anterior regions, such as the raINS and the FP, but not in the ACC. These findings suggest that the raINS plays a key role in long-term abstinence, potentially controlling activation of other areas, such as ACC, related to craving urge itself. The observation that raINS activation was positively correlated with pack-year smoking history (indicative of the accumulated nicotine dose and also possibly the extent of cueconditioning between cigarettes and nicotine) underlines the physiologic importance of the raINS in long-term nicotine abstinence in ex-smokers. In other words, larger 


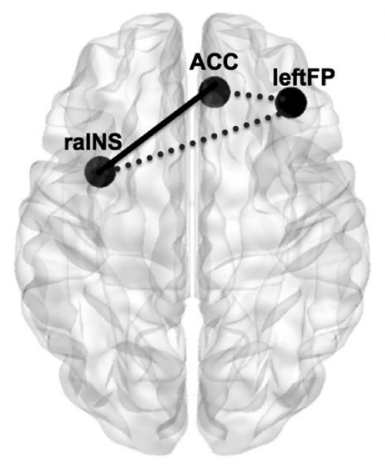

\section{DIFFERENCE IN CONNECTIVITY raINS - ACC}

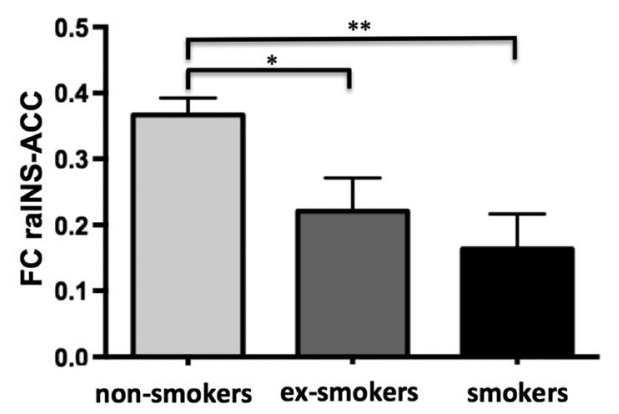

Figure 4 Functional connectivity between the three key regions. All three regions [right anterior insula (ralNS), anterior cingulate cortex (ACC) and left frontal pole] were significantly connected in all three groups. The comparison between ex-smokers versus active smokers and controls revealed that ex-smokers $(P<0.05)$ and active smokers $(P<0.0 \mathrm{I})$ had significantly reduced connectivity between ralNS and ACC, while there was no significant difference between ex-smokers and active smokers. The barplots indicate mean values with SE. $* P<0.05, * * P<0.01$

nicotine exposure is associated with increased activation in the raINS, which in turn regulates the ACC. These findings in ex-smokers suggest a mechanism by which cue-induced brain activation leads to risk of relapse even after prolonged abstinence.

Our second finding relates to alterations in functional connectivity between previously identified brain regions. Specifically, we examined the regions raINS, ACC and left FP, which we identified in the task-related results of the present investigation, and which are in agreement with a previous study on ex-smokers (Nestor et al. 2011). Functional connectivity analysis for these regions revealed significant group differences for both ex-smokers and active smokers when compared with non-smokers, in terms of decreased functional connectivity between raINS and ACC. We found possible evidence of the involvement in these regions as part of a pathway related to craving regulation, i.e. functional connectivity between regulation-related and craving-related brain regions, is disrupted during nicotine addiction and has a persistent effect even after long-term abstinence in exsmokers. We would like to emphasize that we cannot formally exclude a possible alternative explanation that these alterations are a trait of people who smoke, as opposed to people who do not smoke. These connectivity results are in line with a recent study showing decreased connectivity in active smokers versus non-smokers between dorsolateral prefrontal cortex and parietal nodes that are part of the executive control network (ECN) (Weiland et al. 2014) and another study which found decreased global efficiency (a measure that takes into account connectivity) in heavy smokers compared with non-smokers (Lin et al. 2014). Moreover, our findings are consistent with studies on other forms of addiction, including opioids (Liu et al. 2009; Upadhyay et al. 2010; Ma et al. 2011) and Internet addiction (Hong et al. 2013), where a lower functional connectivity is present in addicted subjects. Our results are, however, in contrast with previous findings showing increased connectivity between prefrontal areas in active smokers (Janes et al. 2012). This discrepancy might be due to the fact that the functional connectivity was identified through independent component analysis of resting-state fMRI in this prior study, while we have studied task-based connectivity driven by the processing of video cues that directly induced craving urge. Most importantly, these other studies assessed active smokers, and (to our knowledge) no previous study to date has investigated brain connectivity during long-term cigarette abstinence in ex-smokers. Interestingly, a recent functional connectivity study in short-term nicotine abstinence of 24 hours found alterations in the coupling between the salience (SN) and default mode (DMN) networks (Lerman et al. 2014). This is remarkable because the ACC is part of $\mathrm{SN}$ and DMN, while the aINS is a part of the SN. Notably, the SN, of which the insula is a major component, facilitates orientation to external versus internal information. Lerman et al. (Lerman et al. 2014) illustrate a fundamental role of $\mathrm{SN}$ in toggling resource allocation between the executive control network and DMN during the different states of brain function associated with smoking abstinence and smoking satiety in heavy smokers in short-term nicotine abstinence. Overall, these findings indicate implications of ACC and aINS in both short-term and long-term nicotine abstinence.

Both of the central study findings suggest that the raINS is the key area involved in promotion of long-term nicotine abstinence through the regulation of cravingrelated areas, notably the ACC. Interestingly, a previous study in stroke patients revealed that those patients with lesions in the insula who quit smoking after lesion onset had a high likelihood of quitting easily, immediately and without relapsing or cravings (Naqvi et al. 2007). In addition, a more recent structural MRI study assessed the 
cortical thickness of the raINS and found a negative association with pack-years, cigarette dependence and urge to smoke (Morales et al. 2014). Other studies have found lower GM density in the insula in smokers (compared with non-smokers) (Gallinat et al. 2006; Fritz et al. 2014), perhaps demonstrating the effect of nicotine and other constituents of tobacco smoke on this brain structure. Similarly, anticipation of nicotine administration (Gloria et al. 2009) and administration itself (Stein et al. 1998; Kobiella et al. 2014) have been found to activate the aINS across fMRI studies, indicating that nicotine intake from smoking may affect insula activity as well.

The importance of the insula in craving and drug seeking is reviewed by Naqvi (Naqvi et al. 2014) showing its three different and even opposite functions of incentive motivational processes that can drive addictive behavior, control processes that can inhibit and moderate addictive behavior and interoceptive processes that represent bodily states associated with drug use. The current investigation that indicates the raINS as being the key brain area for craving regulation and inhibition in long-term ex-smokers extends previous studies on short-term nicotine abstinence (Wilson et al. 2005; Wang et al. 2007; Kober et al. 2010). Taken together, our findings add a long-term perspective and temporal continuum; i.e. first, during short-term nicotine abstinence, the raINS is suggested to be responsible for craving regulation, as showed by previous studies and second, during long-term smoking cessation, our results indicate that it promotes abstinence through the enhancement and the inhibition of areas related to craving, in particular, modulating ACC activity.

\section{Strengths and limitations}

In our study, the results of the rating scores analysis confirm that the experimental paradigm induces more craving during cigarette-related than neutral videos and that smokers have the highest induced craving perception compared with the other groups, while the exsmokers can be considered in an intermediate position between smokers and non-smokers.

Nevertheless, it is important to note that some limitations are present and that they can influence the interpretation of the results. First, the number of participants in each group is relatively small, and we did not employ a structured clinical interview for DSM-IV Axis I diagnoses during the participants recruitment to form the different groups, although participant report of smoking behavior is generally reliable (Hendricks et al. 2008). In addition, even though smokers could smoke ad libitum (up until 15 minutes) prior to testing, withdrawal from cigarettes and cigarette craving may have had an effect on the task, and consequently, on our results.
Furthermore, the functional connectivity in our study is conditioned by the presentation of smoking videos stimulations, so the results reflect a connectivity driven by the experimental paradigm, and they may not be comparable with the results of other studies that used resting-state connectivity measures. Moreover, we did not perform a biochemical confirmation of smoking status but relied on the statements of the participants. Finally, it is important to underline possible confounding results from structural changes in WM or GM. To avoid this, VBM and TBSS analyses were conducted, and no differences were observed between the three groups excluding this potential confound. The absence of structural alterations in our study conflicts with previous studies in heavy smokers (Yu, Zhao, \& Lu 2011) or in a much larger group size (Fritz et al. 2014). This discrepancy may indicate that smoking-related structural alterations are relatively small and more pronounced in heavy smokers, explaining the absence of significant differences in our relatively small sample size of ex-smokers and comparably less heavy smokers.

\section{CONCLUSION}

Ex-smokers with long-term smoking abstinence have persistent and dose-dependent changes in brain activity and connectivity, pointing to the key role of the aINS and ACC. By studying a representative sample of ex-smokers, our findings suggest a mechanism underlying the promotion of long-term cigarette abstinence. The evidence of this work can be useful to guide future investigations and psychopharmacological interventions to support patient attempts to quit smoking.

\section{Acknowledgements}

We want to thank all volunteers for their participation in the study. We also thank Prof. Dr. Christian Lüscher for his constructive help during the manuscript preparation. The study was supported by Swiss National Foundation grants SNF project 320030_147126/1, SNF project 320030_127079/1 and SNF PP0OP2-146318 as well as the Fonds Startup du Département de Radiologie et Informatique Médicale in Geneva, Switzerland. Dr. Brody is supported by grants from the National Institute on Drug Abuse (R01 DA20872), the Department of Veterans Affairs, Office of Research and Development (CSR\&D Merit Review Award I01 CX000412) and the Tobacco-Related Disease Research Program (no. 23XT0002).

\section{Disclosure/Conflict of Interest}

The authors declare no conflicts of interest. 


\section{References}

Azizian A, Nestor LJ, Payer D, Monterosso JR, Brody AL, London ED (2010) Smoking reduces conflict-related anterior cingulate activity in abstinent cigarette smokers performing a Stroop task. Neuropsychopharmacol Off Publ Am Coll Neuropsychopharmacol 35:775-782.

Brody AL, Mandelkern MA, Olmstead RE, Jou J, Tiongson E, Allen V, Scheibal D, London ED, Monterosso JR, Tiffany ST, Korb A, Gan JJ, Cohen MS (2007) Neural substrates of resisting craving during cigarette cue exposure. Biol Psychiatry 62:642-651.

Carroll AJ, Sutherland MT, Salmeron BJ, Ross TJ, Stein EA (2013) Greater externalizing personality traits predict less error-related insula and anterior cingulate cortex activity in acutely abstinent cigarette smokers. Addict Biol DOI: 10.1111/adb.12118.

Culbertson CS, Bramen J, Cohen MS, London ED, Olmstead RE, Gan JJ, Costello MR, Shulenberger S, Mandelkern MA, Brody AL (2011) Effect of bupropion treatment on brain activation induced by cigarette-related cues in smokers. Arch Gen Psychiatry 68:505-515.

DeBry SC, Tiffany ST (2008) Tobacco-induced neurotoxicity of adolescent cognitive development (TINACD): a proposed model for the development of impulsivity in nicotine dependence. Nicotine Tob Res Off J Soc Res Nicotine Tob 10:11-25.

Dijk Van KRA, Sabuncu MR, Buckner RL (2012) The influence of head motion on intrinsic functional connectivity MRI. Neuroimage 59:431-438.

Ding X, Lee S-W (2013) Changes of functional and effective connectivity in smoking replenishment on deprived heavy smokers: a resting-state FMRI study. PLoS One 8:e59331.

European Union Campaign 2013 n.d. Ex-smokers Are Unstoppable. Fritz H-C, Wittfeld K, Schmidt CO, Domin M, Grabe HJ, Hegenscheid K, Hosten N, Lotze M (2014) Current smoking and reduced gray matter volume- a voxel-based morphometry study. Neuropsychopharmacol Off Publ Am Coll Neuropsychopharmacol 39:2594-2600.

Gallinat J, Meisenzahl E, Jacobsen LK, Kalus P, Bierbrauer J, Kienast T, Witthaus H, Leopold K, Seifert F, Schubert F, Staedtgen M (2006) Smoking and structural brain deficits: a volumetric MR investigation. Eur J Neurosci 24:1744-1750.

Geier CF, Sweitzer MM, Denlinger R, Sparacino G, Donny EC (2014) Abstinent adult daily smokers show reduced anticipatory but elevated saccade-related brain responses during a rewarded antisaccade task. Psychiatry Res 223:140-147.

George O, Koob GF (2013) Control of craving by the prefrontal cortex. Proc Natl Acad Sci U S A 110:4165-4166.

Gloria R, Angelos L, Schaefer HS, Davis JM, Majeskie M, Richmond BS, Curtin JJ, Davidson RJ, Baker TB (2009) An fMRI investigation of the impact of withdrawal on regional brain activity during nicotine anticipation. Psychophysiology 46:681-693.

Gualano MR, Passi S, Bert F, Torre La G, Scaioli G, Siliquini R (2014) Electronic cigarettes: assessing the efficacy and the adverse effects through a systematic review of published studies. J Public Health Oxf Engl DOI: 10.1093/pubmed/fdu055.

Hendricks PS, Prochaska JJ, Humfleet GL, Hall SM (2008) Evaluating the validities of different DSM-IV-based conceptual constructs of tobacco dependence. Addiction 103:1215-1223.

Holmes S, Zwar N, Jiménez-Ruiz CA, Ryan PJ, Browning D, Bergmann L, Johnston JA (2004) Bupropion as an aid to smoking cessation: a review of real-life effectiveness. Int J Clin Pract 58:285-291.
Hong S-B, Zalesky A, Cocchi L, Fornito A, Choi E-J, Kim H-H, Suh J-E, Kim C-D, Kim J-W, Yi S-H (2013) Decreased functional brain connectivity in adolescents with internet addiction. PLoS One 8:e57831.

Hughes JR, Lesmes GR, Hatsukami DK, Richmond RL, Lichtenstein E, Jorenby DE, Broughton JO, Fortmann SP, Leischow SJ, McKenna JP, Rennard SI, Wadland WC, Heatley SA (1999) Are higher doses of nicotine replacement more effective for smoking cessation? Nicotine Tob Res Off J Soc Res Nicotine Tob 1:169-174.

Hurt RD, Sachs DP, Glover ED, Offord KP, Johnston JA, Dale LC, Khayrallah MA, Schroeder DR, Glover PN, Sullivan CR, Croghan IT, Sullivan PM (1997) A comparison of sustainedrelease bupropion and placebo for smoking cessation. $\mathrm{N}$ Engl J Med 337:1195-1202.

Janes AC, Nickerson LD, Frederick BDB, Kaufman MJ (2012) Prefrontal and limbic resting state brain network functional connectivity differs between nicotine-dependent smokers and non-smoking controls. Drug Alcohol Depend 125:252-259.

Jenkinson M, Bannister P, Brady M, Smith S (2002) Improved optimization for the robust and accurate linear registration and motion correction of brain images. Neuroimage 17:825-841.

Jorenby DE, Leischow SJ, Nides MA, Rennard SI, Johnston JA, Hughes AR, Smith SS, Muramoto ML, Daughton DM, Doan K, Fiore MC, Baker TB (1999) A controlled trial of sustainedrelease bupropion, a nicotine patch, or both for smoking cessation. N Engl J Med 340:685-691.

Killen JD, Fortmann SP, Davis L, Strausberg L, Varady A (1999) Do heavy smokers benefit from higher dose nicotine patch therapy? Exp Clin Psychopharmacol 7:226-233.

Kober H, Mende-Siedlecki P, Kross EF, Weber J, Mischel W, Hart CL, Ochsner KN (2010) Prefrontal-striatal pathway underlies cognitive regulation of craving. Proc Natl Acad Sci U S A 107:14811-14816.

Kobiella A, Ripke S, Kroemer NB, Vollmert C, Vollstädt-Klein S, Ulshöfer DE, Smolka MN (2014) Acute and chronic nicotine effects on behaviour and brain activation during intertemporal decision making. Addict Biol 19:918-930.

Lerman C, Gu H, Loughead J, Ruparel K, Yang Y, Stein EA (2014) Large-scale brain network coupling predicts acute nicotine abstinence effects on craving and cognitive function. JAMA Psychiatry 71:523-530.

Lin F, Wu G, Zhu L, Lei H (2014) Altered brain functional networks in heavy smokers. Addict Biol DOI: 10.1111/ adb. 12155 .

Liu J, Liang J, Qin W, Tian J, Yuan K, Bai L, Zhang Y, Wang W, Wang Y, Li Q, Zhao L, Lu L, von Deneen KM, Liu Y, Gold MS (2009) Dysfunctional connectivity patterns in chronic heroin users: an fMRI study. Neurosci Lett 460:72-77.

Luigjes J, van den Brink W, Feenstra M, van den Munckhof P, Schuurman PR, Schippers R, Mazaheri A, De Vries TJ, Denys D (2012) Deep brain stimulation in addiction: a review of potential brain targets. Mol Psychiatry 17:572-583.

Ma N, Liu Y, Fu X-M, Li N, Wang C-X, Zhang H, Qian R-B, Xu H-S, Hu X, Zhang D-R (2011) Abnormal brain default-mode network functional connectivity in drug addicts. PLoS One 6:e16560.

Morales AM, Ghahremani D, Kohno M, Hellemann GS, London ED (2014) Cigarette exposure, dependence, and craving are related to insula thickness in young adult smokers. Neuropsychopharmacol Off Publ Am Coll Neuropsychopharmacol 39:1816-1822.

Naqvi NH, Rudrauf D, Damasio H, Bechara A (2007) Damage to the insula disrupts addiction to cigarette smoking. Science 315:531-534. 
Naqvi NH, Gaznick N, Tranel D, Bechara A (2014) The insula: a critical neural substrate for craving and drug seeking under conflict and risk. Ann N Y Acad Sci 1316:53-70.

Nestor L, McCabe E, Jones J, Clancy L, Garavan H (2011) Differences in "bottom-up" and "top-down" neural activity in current and former cigarette smokers: evidence for neural substrates which may promote nicotine abstinence through increased cognitive control. Neuroimage 56:2258-2275.

OECD (2012a) OECD Health Data 2012, Accessed on September 2014. Available at: www.oecd.org/health/healthdata.

Polosa R, Caponnetto P, Morjaria JB, Papale G, Campagna D, Russo C (2011) Effect of an electronic nicotine delivery device (e-cigarette) on smoking reduction and cessation: a prospective 6-month pilot study. BMC Public Health 11:786.

Power JD, Barnes KA, Snyder AZ, Schlaggar BL, Petersen SE (2012) Spurious but systematic correlations in functional connectivity MRI networks arise from subject motion. Neuroimage 59:2142-2154.

Smith S, Brady JM (1997) SUSAN—a new approach to low level image processing. Int J Comput Vis 23:45-78.

Smith SM, Jenkinson M, Woolrich MW, Beckmann CF, Behrens TEJ, Johansen-Berg H, Bannister PR, De Luca M, Drobnjak I, Flitney DE, Niazy RK, Saunders J, Vickers J, Zhang Y, De Stefano N, Brady JM, Matthews PM (2004) Advances in functional and structural MR image analysis and implementation as FSL. Neuroimage 23:S208-219.

Smith SM, Jenkinson M, Johansen-Berg H, Rueckert D, Nichols TE, Mackay CE, Watkins KE, Ciccarelli O, Cader MZ, Matthews PM, Behrens TEJ (2006) Tract-based spatial statistics: voxelwise analysis of multi-subject diffusion data. Neuroimage 31:1487-1505.

Smith SM, Johansen-Berg H, Jenkinson M, Rueckert D, Nichols TE, Miller KL, Robson MD, Jones DK, Klein JC, Bartsch AJ, Behrens TEJ (2007) Acquisition and voxelwise analysis of multi-subject diffusion data with tract-based spatial statistics. Nat Protoc 2:499-503.

Stein EA, Pankiewicz J, Harsch HH, Cho JK, Fuller SA, Hoffmann RG, Hawkins M, Rao SM, Bandettini PA, Bloom AS (1998) Nicotine-induced limbic cortical activation in the human brain: a functional MRI study. Am J Psychiatry 155:1009-1015.

Sweitzer MM, Geier CF, Joel DL, McGurrin P, Denlinger RL, Forbes EE, Donny EC (2013) Dissociated effects of anticipating smoking versus monetary reward in the caudate as a function of smoking abstinence. Biol Psychiatry 76:681-688.
Upadhyay J, Maleki N, Potter J, Elman I, Rudrauf D, Knudsen J, Wallin D, Pendse G, McDonald L, Griffin M, Anderson J, Nutile L, Renshaw P, Weiss R, Becerra L, Borsook D (2010) Alterations in brain structure and functional connectivity in prescription opioid-dependent patients. Brain J Neurol 133: 2098-2114.

Wang Z, Faith M, Patterson F, Tang K, Kerrin K, Wileyto EP, Detre JA, Lerman C (2007) Neural substrates of abstinenceinduced cigarette cravings in chronic smokers. J Neurosci Off J Soc Neurosci 27:14035-14040.

Weiland BJ, Sabbineni A, Calhoun VD, Welsh RC, Hutchison KE (2014) Reduced executive and default network functional connectivity in cigarette smokers. Hum Brain Mapp DOI: 10.1002/hbm.22672.

Wilson SJ, Sayette MA, Delgado MR, Fiez JA (2005) Instructed smoking expectancy modulates cue-elicited neural activity: a preliminary study. Nicotine Tob Res Off J Soc Res Nicotine Tob 7:637-645.

Winkler AM, Ridgway GR, Webster MA, Smith SM, Nichols TE (2014) Permutation inference for the general linear model. Neuroimage 92:381-397.

Yu R, Zhao L, Lu L (2011) Regional grey and white matter changes in heavy male smokers. PLoS One 6:e27440.

\section{SUPPORTING INFORMATION}

Additional Supporting Information may be found in the online version of this article at the publisher's web-site:

Table S1 illustrates the list of activation clusters for the GLM analysis of task-related activation illustrated in Fig. 2. Cluster index, number of voxels in cluster, maximum P-value, location of maximum voxel (MAX) and center of gravity of the activation cluster (COG), contrast of parameter estimate (COPE), side $(\mathrm{R}=$ right, $\mathrm{L}=$ left, and $\mathrm{B}=$ bilateral) and anatomic region.

Supplementary Figure A illustrates the opposite comparisons with respect to Fig. 2. There were no supra-threshold activations for ex-smokers versus smokers (A), ex-smokers versus non-smokers (B) or smokers versus non-smokers (C). 